\title{
Aplicación de un Adhesivo Autograbante y uso de la Técnica Incremental Oblicua para Resinas Compuestas en Restauraciones Directas en el Sector Posterior. Reporte de Caso
}

Application ofaself-etchAdhesiveanduseoftheobliqueincremental techniqueforposteriordirectcompositeresinsrestorations. CaseReport

\section{Resumen}

Actualmente contamos con diversos materiales para realizar restauraciones directas en el sector posterior como son los adhesivos de séptima generación y las resinas con nanotecnología. Así como los materiales van variando en el tiempo sucede lo mismo con las técnicas y protocolos a utilizar. En el presente artículo se detalla el protocolo de aplicación de adhesivos de séptima generación como agente de enlace y la aplicación de una resina compuesta utilizando la técnica incremental oblicua en cavidades con y sin ionómero de vidrio en la resolución de un caso clínico. Resultados: Los resultados obtenidos fueron satisfactorios para el paciente ya que se pudo restaurar adecuadamente la pieza dentaria involucrada devolviendo la estética y la función perdida. Conclusiones: El conocimiento profundo de los materiales a utilizar así como las técnicas nos va a llevar a optimizar los resultados de nuestros tratamientos restauradores.

Palabras Clave: Clorhexidina, metaloproteinasas de la matriz, adhesivos, resinas compuestas.

\begin{abstract}
Nowadays we possess diverse materials to realize posterior direct restorations as the seventh-generation adhesives and the nanotechnology resins systems. As well as the materials are changing in the time the same happens with the techniques and protocols to use. In the present article there is detailed the protocol of application of seventh-generation adhesives as agent of link and the application of a composite resin using the oblique incremental technique in cavities with and without glass ionomer in the resolution of a clinical case. Results: The obtained results were satisfactory for the patient since it was possible to restore adequately the dental piece involved returning the aesthetics and the lost function. Conclusions: The deep knowledge of the materials to be used as well as the techniques it is going to lead to optimizing of our rehabilitation treatment.
\end{abstract}

Keywords: Chlorhexidine, matrix metalloproteinases, adhesives, composites resins.

\section{Introducción}

El éxito en la realización de cualquier tratamiento odontológico radica en un adecuado diagnóstico y planificación del mismo, de esta manera se logran optimizar los resultados para el beneficio de los pacientes. ${ }^{1,2}$

Las resinas compuestas como material restaurador directo cumplen con los requisitos de simplificación de los diseños cavitarios, preservación de la estructura dentaria, simplicidad de la técnica a aplicar, multiplicidad de matices y viscosidades; y sobretodo proveer una estética bastante adecuada para restauraciones tanto anteriores como posteriores. ${ }^{3-5}$

Las restauraciones directas con resinas compuestas ofrecen una alternativa de tratamiento estética y funcional frente a otras restauraciones convencionales como incrustaciones o coronas cerámicas, pues preservamos mayor cantidad de estructura dentaria, reducimos el
Casos Clínicos

\section{César Lamas Lara', Giselle Angulo de la Vega' ${ }^{2}$, Sergio Alvarado-Menacho ${ }^{3}$}

1 Cirujano Dentista, Diplomado en Odontología Restauradora y Estética de la Facultad de Odontología de la UNMSM. Docente del Curso de Operatoria Dental I y II ULADECH Católica.

2 Cirujano Dentista, Estudios de Especialidad de Rehabilitación Oral de la Facultad de Odontología de la UNMSM.

3 Profesor del Departamento Académico de Estomatología Rehabilitador de la Facultad de Odontología de la UNMSM.

Correspondencia:

CD César Lamas Lara

Santa Honorata 415. Urb. Pando, 3era Etapa. San Miguel. Lima 1. Perú.

Teléfono: 999491403

e-mail: cesar2579@hotmail.com

Fecha de recepción: 08 de abril

Fecha de aceptación: 14 de junio número de citas clínicas y costos para el paciente. ${ }^{6-8}$

El profundo conocimiento de los materiales y las técnicas a utilizar van a facilitar la ejecución de un tratamiento satisfactorio. Cabe destacar que no todas las resinas compuestas se comportan de la misma manera, tanto en sus propiedades físicas y mecánicas como en su manipulación. No es suficiente realizar una restauración visualmente correcta si esta no cumple su función dentro del sistema estomatognático. ${ }^{\text {y } 10}$ 
Los sistemas adhesivos han sido clasificados de diferentes maneras siendo la clasificación por generaciones la más utilizada. En la actualidad podemos clasificarlos de acuerdo a las estrategias de unión a los estratos dentarios, y así se clasificarían en adhesivos convencionales (con la aplicación de ácido grabador), adhesivos autograbadores y materiales autoadhesivos. ${ }^{11 \text { y } 12}$

El uso de adhesivos autograbantes es cada vez más frecuente por la practicidad de la técnica que conlleva a la presencia de menos errores. En este tipo de adhesivos la aplicación de ácido fosfórico al $37 \%$ por 15 segundos sobre la superficie del esmalte es recomendado por la mayoría de los fabricantes, esto nos puede garantizar un sellado marginal altamente confiable en las restauraciones. ${ }^{13}$ y 14

La clorhexidina usada en operatoria dental tiene un efecto antibacteriano y es un inhibidor de la actividad proteolítica de las Metaloproteinasas de la Matriz (MMPs). Estas MMPs intervienen en los procesos de formación del diente y también en la presencia de caries dental degradando la matriz extracelular. Al aplicar clorhexidina sobre la dentina en el procedimiento adhesivo se puede inhibir la degradación de la capa hibrida garantizando un mayor longevidad de la interfase resina-dentina. ${ }^{15-17}$

El objetivo del caso fue devolver al paciente una adecuada estética y función masticatoria con el uso de materiales restauradores directos.

\section{Reporte del caso}

Paciente de sexo femenino de 24 años de edad que acude a la consulta clínica expresando el deseo de realizar el cambio de restauraciones antiguas realizadas hace 10 ańos.

$\mathrm{Al}$ examinar a la paciente se puede apreciar restauraciones con amalgama a nivel de la pieza $3.6(\mathrm{O})(\mathrm{V})$. (Fig. 1)

\section{Diagnóstico}

Del estado de salud general: Paciente con aparente estado de salud general, sin riesgo sistémico al tratamiento estomatológico.

Del estado de salud estomatológico: $\mathrm{Pa}-$ ciente con:

1 Caries dental: presencia de restauración defectuosa y caries recidivante en la piezas $3.6(\mathrm{O})(\mathrm{V})$.

\section{Plan de Tratamiento}

1 Fisioterapia oral, enseñanza de la técnica de cepillado, hilo dental y enjuague bucal.

2 Restauraciones directas con resina compuesta en piezas $3.6(\mathrm{O})(\mathrm{V})$.

\section{Tratamiento}

Se procedió a realizar el retiro de la restauración de amalgama y la eliminación del tejido cariado con aislamiento absoluto, se seleccionó el color (Fig. 2).

Examinando las necesidades de cada cavidad se decidió utilizar como base cavitaria en la cavidad vestibular ionómero de vidrio y en la cavidad oclusal no se aplicó ningún protector debido a que esta era una cavidad de una profundidad de $2 \mathrm{~mm}$ y se consideró que no lo requiere.

Una vez realizada la cavidad procedemos a aplicar ácido fosfórico al $37 \%$ en gel solamente sobre el esmalte por 15 segundos (Fig. 3). Lavamos con abundante agua y secamos con papel absorbente (Fig. 4).

Para inhibir la acción de las metaloproteinasas aplicamos clorhexidina al 2\% por 15 segundos en toda la cavidad, lavamos con abundante agua y secamos con papel absorvente sin resecar.

Posteriormente se aplicó ionómero de vidrio modificado con resina como base cavitaria en la cavidad vestibular debido a su profundidad (Fig. 5) y se fotopolimerizó 20 segundos. Al aplicar ionómero de vidrio después del grabado con ácido fosfórico, evitamos hidrolizar y deteriorar su superficie ya que esto podría afectar la unión con la resina compuesta. Como se utilizó un ionómero modificado con resina no hay la necesidad de realizar un grabado ácido sobre el ionómero ya que el componente resinoso del ionómero permitirá la unión correcta con el adhesivo.

Inmediatamente se aplicó dos capas de un sistema adhesivo de séptima generación en la cavidad oclusal y vestibular y se fotopolimerizó 10 segundos según las indicaciones del fabricante. (Fig. 6)

Una vez aplicado el adhesivo se procedió a realizar la aplicación de los incrementos de resina compuesta utilizando la técnica incremental oblicua. (Fig. 7 y 8) En la caja oclusal se aplicó los incrementos de resina compuesta realizando cúspide por cúspide colocando el primer incremento desde el surco central hacia la cima de la cúspide distolingual, definiendo la anatomía propia de esa cúspide, luego procedemos a fotopolimerizar el incremento por 20 segundos. De la misma manera realizamos las demás cúspides hasta completar la anatomía de la caja oclusal. En la caja vestibular realizamos la misma técnica tratando de que cada incremento de resina este en contacto con 2 paredes para reducir el factor $\mathrm{C}$ hasta completar la caja vestibular, de esta manera obtendremos un factor $\mathrm{C}$ de 0.5 que es lo ideal en una cavidad posterior.

Luego de aplicado el último incremento de resina cubrimos toda la restauración con glicerina líquida con la finalidad de que la capa inhibida por el oxígeno no se forme y obtener una polimerización completa del material (Fig. 9). Retiramos el aislamiento absoluto y procedemos a hacer el control de la oclusión con papel de articular (Fig. 10). Finalmente realizamos el acabado y pulido de nuestras restauraciones con fresas de granos fino y cauchos abrasivos. (Fig. 11)

Se utilizó una resina compuesta de color A2 con nanotecnología por presentar buenas características tanto mecánicas como ópticas.

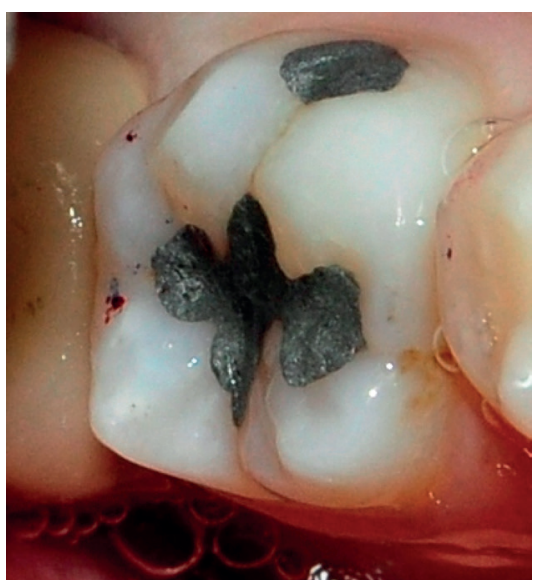

Fig. 1. Vista Inicial.

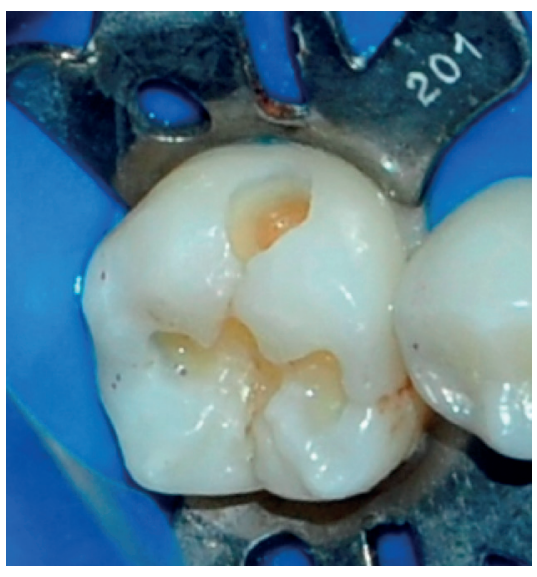

Fig. 2. Retiro de la amalgama y eliminación de la caries. 


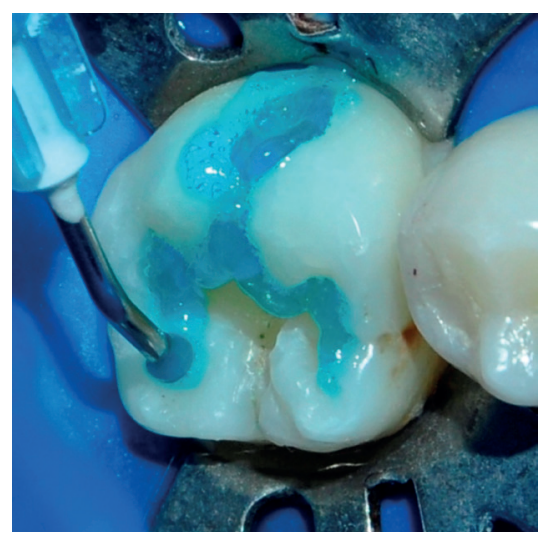

Fig. 3. Aplicación del ácido fosfórico en esmalte.

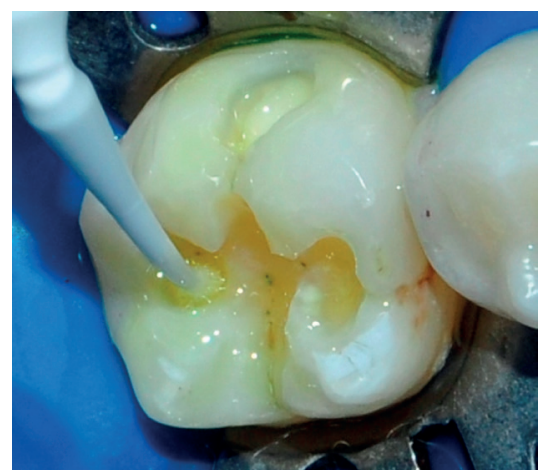

Fig. 6. Aplicación del adhesivo autograbante.

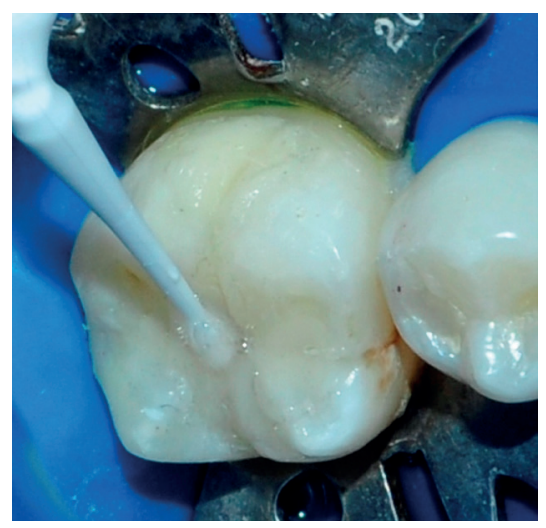

Fig. 9. Aplicación de glicerina líquida en la superficie de la restauración.

\section{Resultados}

Los resultados obtenidos fueron satisfactorios para la paciente ya que se logró devolver una morfología y estética satisfactoria devolviendo así la función de la pieza dentaria.

\section{Discusión}

La aplicación de adhesivos de séptima generación se está popularizando cada vez más y esto se debe a su practicidad y simplicidad en su aplicación sin

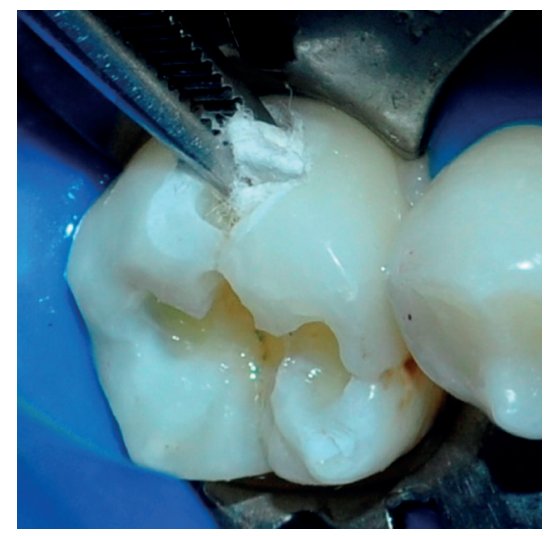

Fig. 4. Secado de superficies.

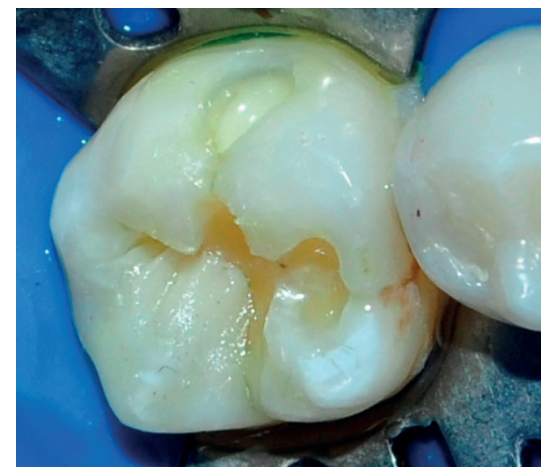

Fig. 7. Aplicación de los incrementos de resina compuesta.

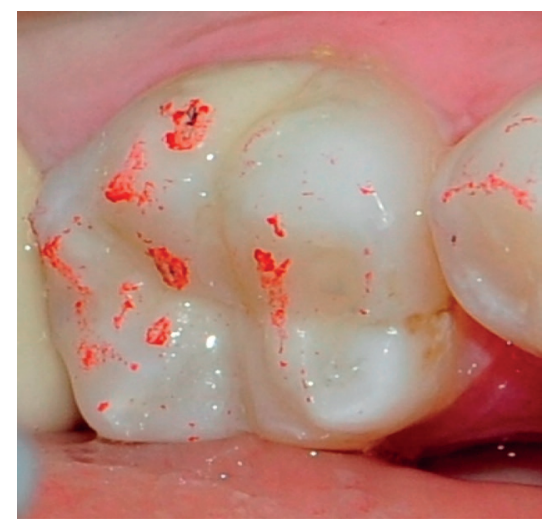

Fig. 10. Control de la oclusión.

embargo el aplicar ácido grabador con esto adhesivos parece ser una sugerencia valida al menos en esmalte por mejorar la interfase de unión resina-dentina, garantizando de esta manera la longevidad de nuestras restauraciones. ${ }^{18 \text { y } 19}$

La aplicación de la clorhexidina para mejorar la longevidad de la interfase diente-restauración todavía esta en discusión en relación a los adhesivos de séptima generación ya que no se cuentan con estudios que avalen su uso pero si existen estudios en relacionados a los adhesivos de quinta generación en los

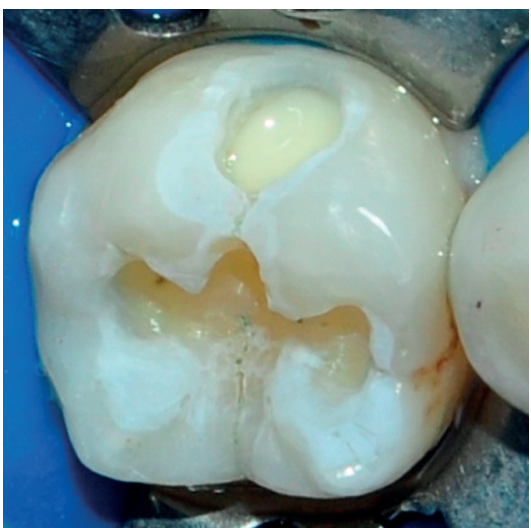

Fig. 5. Aplicación de ionómero como base en la cavidad vestibular.

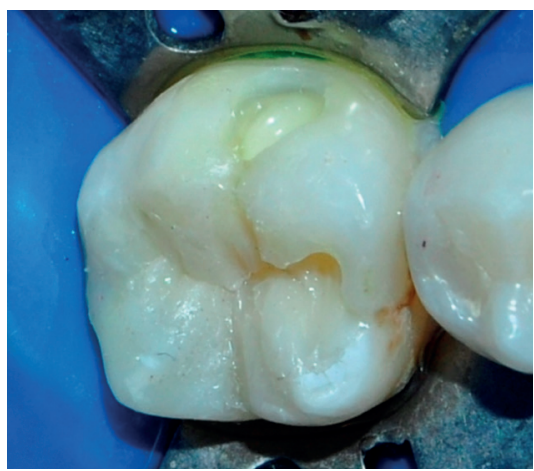

Fig. 8. Aplicación de los incrementos de resina compuesta.

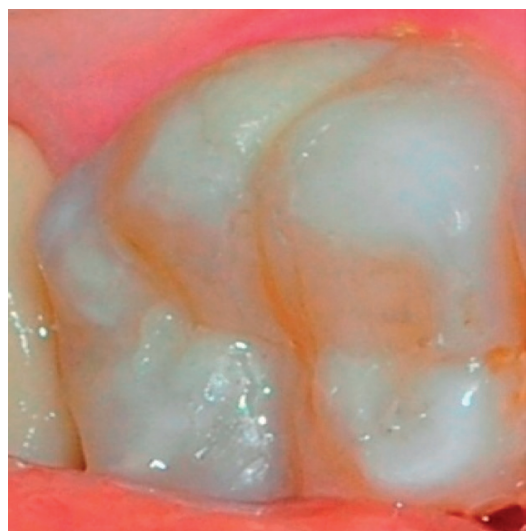

Fig. 11. Vista final de la restauración.

que se logra demostrar los beneficios de su aplicación en la conservación de la capa hibrida inhibiendo la acción de las MMPs y estos conceptos se pueden extrapolar a los adhesivos de séptima generación. ${ }^{20 \text { y } 21}$

Las restauraciones directas con resinas compuestas es una alternativa que nos proporciona buenos resultados tanto estéticos como funcionales siempre $\mathrm{y}$ cuando dominemos a cabalidad los protocolos y técnicas para su aplicación. La técnica incremental oblicua para la aplicación de resinas compues- 
tas tiene el objetivo de reducir el estrés de contracción del material obteniendo un valor de 0.5 que es aceptable para el sector posterior; otra alternativa que podríamos utilizar sería reducir el volumen de resina compuesta empleando materiales como los ionómeros de vidrio, a menor volumen menor contracción. Estos métodos para reducir el estrés no son excluyentes entre si. ${ }^{22,23}$

\section{Conclusiones}

Con el avance de los nuevos materiales restauradores los protocolos restauradores se han ido modificando; esta en cada profesional capacitarse de tal manera que pueda desenvolverse de la mejor manera posible. La aplicación correcta de los materiales restauradores así como una técnica prolija nos garantizara el éxito en nuestros tratamientos restauradores.

El uso de la clorhexidina con la aplicación de adhesivos de séptima generación todavía se encuentra en controversia debido a la ausencia de mayor cantidad de estudios que avalen su aplicación clínica, pero su uso es recomendado por algunos autores por considerarlo importante en la conservación de la unión resina dentina inhibiendo la actividad de las metaloproteinasas de la matriz.

Si bien es cierto los adhesivos de séptima generación aparecieron en el mercado con la finalidad de simplificarnos los pasos clínicos, en la práctica esta simplificación no es tan real, pero si nos garantizan protocolos más seguros al momentos de realizar los procedimientos restauradores.

La técnica incremental oblicua en restauraciones posteriores nos facilita no solamente tratar de controlar la contracción del material sino la posibilidad de realizar una restauración con mayor rapidez y anatomía.

\section{Referencias bibliográficas}

1. Lamas C; Angulo G; Alternativa de Restauración Estética en caso de Discromía, Odontol. Sanmarquina, 2010; 13 (2): 38-41.

2. Lara TL, Ochoa TJ, Gaitán VJ, Herrera CM. Rehabilitación con coronas completas de metal - ce- rámica y prótesis parciales removibles convencionales. Odontol. Sanmarquina 2009; 12(2): 78-82.

3. Albers HF. Tooth colored restorations: principles and techniques. 9th edition. BC Decker Publishing Ontario, Canadá. 2002. 203.

4. Conceição EN et al. Restauraçôes Estéticas. Compósitos, Cerâmicas e Implantes. Ed Artmed. Porto Alegre, Brasil. 2005. 152.

5. Ardu S, Krejci I. Biomimetic direct composite stratification technique for the restoration of anterior teeth. Quintessence Inter 2006; 37(3): 167-74.

6. Magne P, Holz J. Stratification of composite restorations. Systematic and durable replication of natural aesthetics. Pract Periodont Aesthet Dentistry 1996; 8: 61-8.

7. Ardu S, Krejci I. Biomimetic direct composite stratification technique for the restoration of anterior teeth. Quintessence Inter 2006; 37(3): 167-74.

8. Hidalgo RC. Técnica de estratificación por capas naturales a mano alzada con resinas compuestas. Visión Dental 2008; 11 (3): 412418.

9. Lamas C; Angulo G; Reconstrucción del Sector Anterior con Resinas Compuestas, Odontol. Sanmarquina, 2009; 12 (2): 90-92.

10. Lamas C; Angulo G; Técnica de Mock Up y Reconstrucción por Capas Anatómicas, Odontol. Sanmarquina, 2011; 14 (1): 19-21.

11. Van Meerbeek B, Vargas M, Inoue $S$, Yoshida Y, Peumans M, Lambrechts $\mathrm{P}$, et al. Adhesives and cements to promote preservation dentistry. Oper Dent. 2001;26(Suppl 6):119-44.

12. Van Meerbeek B, Peumans M, Poitevin A, Mine A, Van Ende A, Neves A, et al. Relationship between bond-strength tests and clinical outcomes. Dent Mater. 2010;26(2):e100-21.

13. Padrós-Serrat J, MonterrubioBerga M, Padrós-Cruz EEsteban. Adhesivos autograbantes: ¿Grabar o no grabar? RCOE. 2003 Ago; 8(4): 363-375.
14. Soares C; Guimarães C; Freitas P; Soares A. Effects of previous treatments on bond strength of two self-etching adhesive systems to dental substrate. Journal of adhesive dentistry 2007;9:291-296

15. Hannas AR, Pereira JC, Granjeiro JM, Tjäderhane L. The role of matrix metalloproteinases in the oral environment. Acta Odontol Scand. 2007;65(1):1-13.

16. Hebling J, Pashley DH, Tjäderhane L, Tay FR. Chlorhexidine arrests subclinical degradation of dentin hybrid layers in vivo. J Dent Res. 2005;84(8):741-6. Erratum in: J Dent Res. 2006;85(4):384.

17. Breschi L, Mazzoni A, Ruggeri A, Cadenaro M, Di Lenarda R, De Stefano Dorigo E. Dental adhesión review: aging and stability of the bonded interface. Dent Mater. 2008; 24(1): 90-101.

18. Van Landuyt KL; Kanumilli; De Munck J; Peumans M; Lambretchs P; Van Meerbeek B. Bond strenght of a mild self etch adhesive with and without prior acid etching. Journal of Dentistry 2006 34, 77 85.

19. Miguez P; Castro P; Nunes M; Walter R; Pereira P. Effect of Acid etching on the enamel bond of two self etching systems. Journal of adhesive dentistry 2003; 5: 107-112.

20. Brackett MG, Tay FR, Brackett WW, Dib A, Dipp FA, Mai S, et al. In vivo chlorhexidine stabilization of hybrid layers of an acetonebased dentin adhesive. Oper Dent. 2009; 34(4): 379-83.

21. Ricci HA, Sanabe ME, de Souza Costa CA, Pashley DH, Hebling J. Chlorhexidine increases the longevity of in vivo resin-dentin bonds. Eur J Oral Sci. 2010; 118(4): 4116.

22. Davidson CL. Glass ionomer bases under posterior composites. Journal of Aesthetic Dentistry. 1994; 6: 223-6.

23. Feilzer AJ, De Gee AJ, Davidson CL. Curing contraction of composites and glass-ionomer cements. J Prosth. Dent. 1988; 59(3): 297300. 